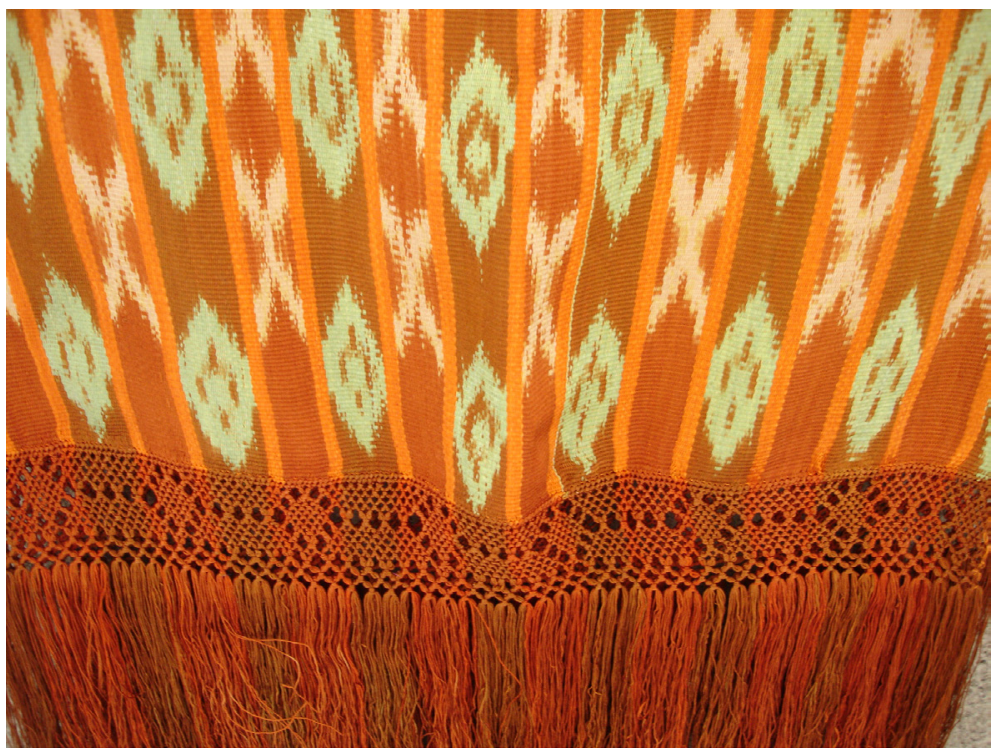

\title{
PATRIMONIO CULTURAL, CAMBIO Y PERMANENCIA
}

\author{
Malo González, Claudio
}

Correspondencia: cmalo@uazuay.edu.ec 


\section{Resumen:}

Los seres humanos somos temporalizados. Las experiencias vitales se dan en el presente sustentadas por el pasado y con proyecciones al futuro. El patrimonio cultural está en el pasado y hay que reconocerlo y salvaguardarlo porque pone de manifiesto los valores de lo que fuimos, como lo demuestran objetos, monumentos y obras de arte. Desde hace pocos años se reconoce como parte del patrimonio cultural manifestaciones populares que, sin ser materiales, se mantienen como parte de la tradición. El patrimonio cultural estructura la identidad de los pueblos que con más fuerza hay que mantenerla para superar el peligro de la globalización. La temporalidad es esencial a este patrimonio cuyo respeto y valorización deben darse en los sectores privados y públicos.

Palabras clave: cultura, patrimonio, cambio, temporalidad. 


\section{CULTURAL HERITAGE, CHANGE AND PERMANENCY}

\section{Abstract:}

Human are temporalized beings. Vital experiences occur in the present, are supported by the past, and project towards the future. Cultural heritage is part of history and must be recognized and safeguarded because it manifests the values of who we once were, as demonstrated by objects, monuments and works of art. Just a few years ago, popular manifestations began to be recognized as part of cultural heritage; without being material, they remain a part of tradition. Cultural heritage structures the identity of the people, which must be maintained with great effort to overcome the danger of globalization. Temporality is essential to this heritage, whose respect and valuation should be a given in the private and public sectors.

Keywords: Culture, heritage, change, temporality. 


\section{Palabras y cultura}

Las palabras tienen vida, aparecieron alguna vez como respuesta a la necesidad humana de trasladar los conceptos a signos fonéticos y gráficos, pero no se convirtieron en clisés inamovibles. La cultura es dinámica por naturaleza y el cambio no es el resultado de una mente prodigiosa. Surgen nuevas situaciones, aparecen nuevos elementos materiales y no materiales que se incorporan a la vida colectiva, y se requiere de nuevas palabras. Complicado y farragoso sería crear una nueva palabra para cada cambio. Palabras ya existentes que tienen vida se acoplan a las innovaciones encontrando similitudes con diferentes niveles de coherencia.

Algunos antropólogos creen que en el tránsito de los simios al ser humano, la capacidad de crear símbolos y palabras fue un hito definitorio. Sin entrar en discusiones de minucias, lo real es que el uso del lenguaje contribuyó al acelerado distanciamiento de nuestra especie de las demás del reino animal, ya que los idiomas constituyen el fundamento de las culturas que superaron al instinto en cuanto puede ser manejado, organizado y manipulado de acuerdo con las motivaciones y voluntades de los diferentes grupos ${ }^{1}$.

El término Patrimonio es un caso de esta vitalidad de las palabras. Necesitamos contar con bienes materiales para hacer frente a las necesidades de la vida; como tenemos el privilegio o la carga de anticipar -no adivinar- el futuro y de organizar nuestro comportamiento considerando lo

1 Un hito de gran importancia en el desarrollo cultural de la humanidad fue la invención para trasladar ideas o sonidos a signos gráficos. 
que puede ocurrir; a diferencia de las aves para las que todo es de ellas sin las ataduras de la propiedad privada, nosotros nos hemos tornado acumuladores de riqueza para planificar lo que nos ocurrirá. Al conjunto de bienes acumulados por una persona se lo denomina patrimonio, que se amplía a la familia y supervive mediante la herencia que rompe los limitantes biológicos de la vida.

El diccionario de la Real Academia de la Lengua define patrimonio como "Hacienda que alguien ha heredado de sus ascendientes. Conjunto de los bienes propios adquiridos por cualquier título". Este concepto se centra en la riqueza individual; pero los seres humanos somos animales sociales, lo que implica que debamos vivir en colectividades, comenzando con la familia. No se limitan a la mera suma de individuos. En una cultura ellos están vinculados mediante realidades de diversa índole, común a los integrantes de la colectividad. Cada cultura, en el sentido antropológico del término, es propietaria de una serie de rasgos ${ }^{2}$. El antropólogo senegalés Amadou Mahtar Mbow la define como:

"Es a la vez aquello que una comunidad ha creado y lo que ha llegado a ser gracias a esa creación; lo que ha producido en todos los dominios donde ejerce su creatividad y el conjunto de rasgos materiales y espirituales que a lo largo de ese proceso, ha llegado a modelar su identidad y distinguirla de otras."

¿A quién pertenece la cultura? A todos y a nadie. A todos los que forman parte de esa colectividad organizada, a nadie en el sentido individual del término propiedad. Mucho de lo que creamos los seres humanos, individual 2 El término propiedad puede ser individual o colectivo, como es el caso de una corporación. La propiedad cultural es diferente en cuanto a su manejo. 
y colectivamente, son objetos transitorios, efímeros en algunos casos, como el pan que en corto tiempo se consume. Otras obras se elaboran con el propósito de que tengan una duración que sobrepase el ciclo vital personal y cumpla sus funciones para bienestar de generaciones futuras, obras materiales como las casas o no materiales como las leyes o piezas literarias. Más allá de los planes e intenciones, en algunos casos dejan de tener importancia en corto tiempo y en otras, algo que fue hecho con una pretensión transitoria mantiene vida por muchísimos años y generaciones ${ }^{3}$.

Ante esta nueva visión la palabra patrimonio supera su contenidoeconómicoyse "culturiza". La propiedad individual conlleva derechos y obligaciones económicas reconocidos y regulados por el tradicional concepto de patrimonio. El patrimonio cultural pertenece a todos, pero no puede ser negociado con la misma soltura que el económico. Los Estados funcionan con leyes, los gobernantes no son propietarios de los países, su meta es lograr y robustecer el bien común que va más allá del monetario y, siendo la cultura parte de esta forma de bien, tiene que regularse su uso, comenzando con el reconocimiento de esta condición y estableciendo limitaciones a la manera de disponer de ellos por parte de los ciudadanos. Aunque legalmente algunos ciudadanos sean propietarios de objetos que reúnen estas características, para disponer de él deben someterse a una serie de normas en las que, más allá de los intereses personales, están los de la colectividad. Un ejemplo esclarecedor es la prohibición de sacarlos al exterior de manera definitiva. Por otra parte. el Estado, que regula su uso tiene que asumir una 3 Una pieza de cerámica precolombina hecha para satisfacer necesidades personales, al ser rescatada mediante la arqueología se convierte en patrimonio cultural. 
serie de obligaciones con relación a estos bienes, como conservarlos adecuadamente para que sus condiciones no sean sustancialmente alteradas y, si es que es necesario, someterlas a un proceso de restauración ${ }^{4}$.

\section{Cultura, patrimonio y tiempo}

La cultura es el resultado de la condición humana, desconozco si alguna especie animal tiene una cultura. La creatividad se pone de manifiesto en un momento dado ante diversas circunstancias y partiendo de múltiples motivaciones. La creatividad no es una respuesta fija como la instintiva ante un estímulo, parte de una planificación, simple o compleja, para hacer frente a situaciones que se dan en el presente y cuyos efectos o resultados se ponen de manifiesto en el futuro. Somos en buena medida lo que la cultura de la que formamos parte nos ha hecho, es decir el pasado es inevitable e irrenunciable en las personas y las colectividades. La decisión de solucionar algún tipo de problema se toma en el presente y sus efectos se valoran en el futuro. La cultura está conformada por una serie de objetos materiales y no materiales, pautas de conducta y creencias que no se agotan de manera inmediata, sino que permanecen en el tiempo superando las limitaciones generacionales y manteniendo su validez para los que existen y existirán en el futuro.

La cultura no se inventa, se forja a lo largo del tiempo y siempre está cambiando conforme a las nuevas condiciones de la vida colectiva. Hay rasgos culturales que dejan de

4 En la restauración hay alteraciones de objetos como pinturas y esculturas con el propósito de que recuperen su condición inicial. 
tener vigencia como los carruajes tirados por caballos para transporte, debido a que los vehículos automotores los han desplazado y quedan como recuerdos de tiempos idos y, a veces, como piezas de museo. Hay otros que se mantienen porque de alguna manera siguen, aunque sea parcialmente, satisfaciendo las necesidades y hay también algunos que, sin cumplir las funciones para las que fueron creados subsisten y son objeto de cuidados y tratamientos especiales porque han adquirido un significado nuevo en la colectividad.

En el ámbito de lo material, se los valora por cuanto testimonian formas de vida y capacidades de épocas anteriores y las personas sienten especial curiosidad por conocer qué funciones tenían porque, de manera expresa o tácita, hay conciencia de nuestra condición temporal. Los objetos que por diferentes razones han sobresalido en la época en que fueron hechos y no se han destruido totalmente, son parte del patrimonio cultural y deben ser conservados y mantenidos ya que, más allá de los hechos y las ideas de la historia, los objetos en sí son más elocuentes para una valoración intelectual y emotiva del pasado ${ }^{5}$.

La conservación de los bienes patrimoniales no se limita al presente, se toman medidas para mantenerlos para las futuras generaciones pues, dada nuestra condición, no podemos prescindir del pasado lejano o cercano, al que debemos respetarle. Pobre en extremo es la visión de unos pocos en el sentido de que, si la vida es cambio, hay que destruir lo que ya no satisface necesidades actuales 5 La trascendencia histórica de personajes transforma en patrimonio objetos de su pertenencia aunque en sí mismos carezcan de importancia, como la pluma que algún célebre escritor usó para escribir sus obras. 
y organizar el comportamiento mirando tan sólo el futuro, pues el pasado ya no es necesario.

François Ost, en su artículo "el Patrimonio y las Generaciones Futuras", publicado en el libro "Claves del Siglo XXI editado por la UNESCO nos dice:

"Mientras antes del patrimonio se preservaba con cuidado para mantener la fe en nuestros antepasados, hoy lo protegemos en consideración a nuestros sucesores, como si fuera el futuro, más que el pasado, lo que hay que salvaguardar.

Por un lado, tenemos las generaciones pasadas, los antepasados remotos que comenzaron a construir el patrimonio $y$, por otro, las generaciones futuras, los imprecisos donatarios de la donación. Entre ellos están las generaciones presentes, agentes efímeros de la transmisión. Si somos capaces de elevarnos al nivel de lo simbólico, es posible invertir la proposición y sostener que es precisamente por medio de su integración en un linaje transhistórico cómo el sujeto deja de ser un individuo aislado. De modo que, con el fin de existir verdaderamente, para salir del ciclo precario de consumo y producción, uno debe pensar en sí mismo como alguien conectado, como el corredor de relevos que es responsable del testigo en las últimas etapas de la carrera" ${ }^{6}$.

Esta cita nos muestra que no cabe, como muchos piensan, limitar el patrimonio al pasado. Las preservaciones y restauraciones, en todas las dimensiones, se las realizan en un presente para que se mantengan en el futuro por tiempo indefinido. De ninguna manera pasado

6 UNESCO 2002, Claves del Siglo XXI, Editorial Crítica, Barcelona. Página 207 
y futuro son contradictorios, se complementan, pues la condición de temporalidad de la especie humana es una. Valoramos al pasado, pero tenemos un compromiso con las generaciones que nos sucederán.

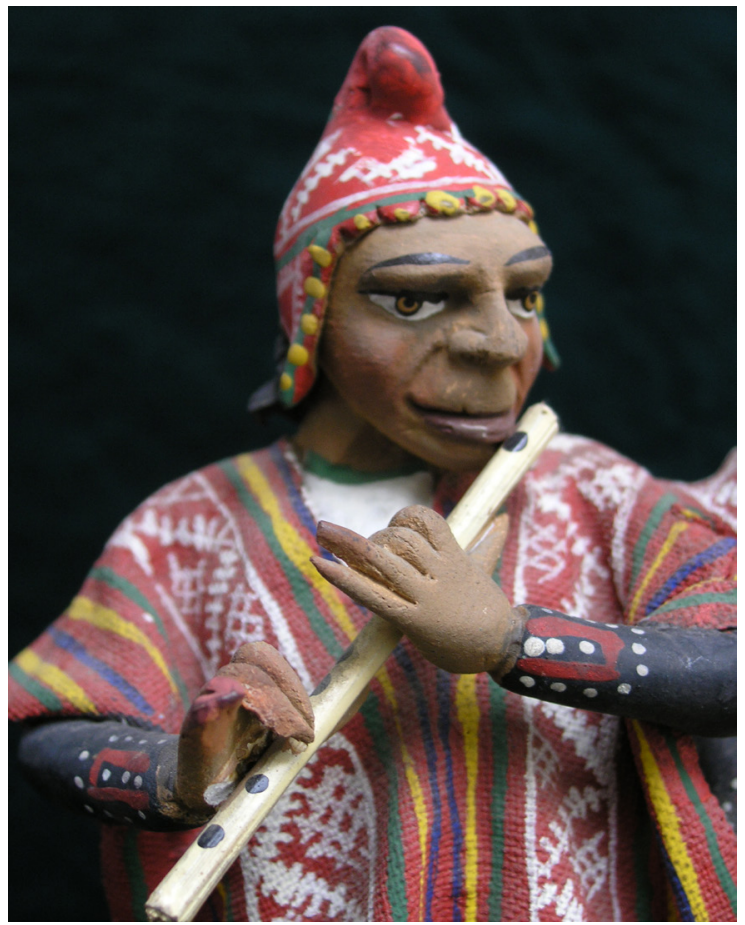

\section{Patrimonio e identidad}

La diversidad es una de las características definitorias del ser humano. Biológicamente las diferencias raciales son reducidas si las comparamos con otras especies animales como la de "nuestros mejores amigos", los perros, entre los que encontramos variaciones que van desde un chiguagua hasta un gran danés. La diversidad fundamental está en el campo de la cultura, resultado de 18 
nuestra creatividad. A diferencia de las demás especies del reino animal cuyo comportamiento se limita a responder a los estímulos que provienen del exterior. Nosotros actuamos, esto es incorporamos a nuestro interior en forma de ideas los fenómenos externos, los analizamos mentalmente y retornamos con iniciativas para realizar cambios. Dada nuestra capacidad de pensar anticipando resultados futuros, resolvemos una misma situación de diversas maneras. Nacemos y nos desarrollamos en una cultura creada por los que nos antecedieron, con capacidad de introducir en su acervo cambios pequeños y grandes. Esta forma de comportamiento se da a nivel individual, pero siendo por naturaleza animales sociales, los cambios pueden afectar a todos los integrantes de la colectividad de la que formamos parte. Esta peculiaridad ha hecho que hayan surgido múltiples culturas diferentes, cada una de ellas integrada por una serie de rasgos ordenados y organizados.

Un buen número de rasgos es igual en todas las culturas, de allí que las diferencias provengan de aquellos que son propios de una de ellas. Estos conglomerados sociales no son entes aislados -como las mónadas en la filosofía de Leibnitz-, se intercomunican permanentemente incorporando rasgos de otras en función de la eficiencia para la satisfacción de necesidades. En algunos casos, ante un problema, las culturas crean internamente la solución que se torna permanente por iniciativa propia, pero lo más frecuente es que mediante la comunicación se incorporen soluciones ya probadas por otras. Uno de los peligros que anota McLuhan del creciente y en apariencia imparable proceso de globalización, es la unificación cultural del mundo ${ }^{7}$ que, por la creciente celeridad en la comunicación,

7 El sociólogo canadiense Marshall McLuhan en 1968 publicó 
se convertiría en una aldea global, lo que para algunos consideran positivo. La mayoría de personas aspira a que su colectividad sea diferente -no sea del montón- mediante la preservación de rasgos que le dan identidad, ya que es parte gratificante de nuestra manera de ser y pensar, ser distintos como individuos e integrantes de una comunidad.

La identidad cultural no se la inventa, conforma en un momento, ni mediante decretos. Es el resultado de un largo proceso que puede durar siglos y que se mantiene debido a una aceptación -en la mayoría de los casos tácita- de la mayor parte de las comunidades ${ }^{8}$. En este sentido la identidad es parte del presente, pero conformada colectivamente en el pasado y con miras a mantenerse en el futuro. Por mucho que algunos traten de modernizarse y cambiar siguiendo el ejemplo de culturas o países a los que consideran más avanzados, no es posible despojarse totalmente de aquello que nos ha hecho. El futuro es cambio, el pasado conservación, pero si queremos cambiar hay que partir de algo que ya existe y se ha estructurado a lo largo del tiempo. Es irreal y precipitado considerar que lo único positivo en la existencia es el cambio, que se lo consigue renunciando al pasado portador de todos los retrasos, como igualmente irreal y precipitado sería creer que la sabiduría sólo está en lo que antes de nosotros se ha hecho y se lo debe mantener sin cambio alguno. Tradición y cambio no son excluyentes ni contradictorios, son parte de la temporalidad humana. Lo deseable sería llegar a un sano equilibrio.

La identidad se fundamenta en la tradición que, superando la deterioradora acción del tiempo se mantiene

su libro Guerra y Paz en la Aldea Global, si bien en obras anteriores ya había utilizado este término.

8 Con frecuencia suele llamársela memoria colectiva.

20 
y nos hace distintos de cultura a cultura. En sentido amplio es parte de todos, propiedad de todos, patrimonio de todos. La historia social analiza estas situaciones y objetos, muchos de los cuales merecen especial respeto por testimoniar hechos de especial importancia en lo que somos. Si la meta fundamental de la historia es entender el pasado en sus múltiples variables, es indispensable que algunos elementos materiales merezcan especial atención, más allá de las características intrínsecas, porque son manifestaciones de formas de vida y visiones que se tenía en el pasado. Las pirámides de Egipto o Machu Picho se caracterizan por sus componentes materiales grandiosos, pero además nos conducen a la comprensión de las cosmovisiones de los egipcios y los incas precolombinos. Además de no destruirlos intencionalmente -como ha ocurrido en algunos casos- es necesario mantenerlos y restaurarlos sin atentar contra su estructura esencial, y cuidándolos ante posibles agresiones de que pueden ser víctimas, creando si es necesario o robusteciendo la conciencia colectiva de su importancia ${ }^{9}$. Si el patrimonio cultural pertenece a todos, los derechos y obligaciones ciudadanos tienen que ser coherentes con esta pertenencia.

\section{Patrimonio cultural inmaterial}

Cuando preguntarnos a personas con diferentes niveles de formación académica qué nos diferencia de los animales, la respuesta más generalizada es que podemos razonar homo sapiens-. Si otra de las características fundamentales para consolidar esta diferencia es la capacidad de elaborar creativamente objetos partiendo de los materiales del

9 El desarrollo de la Arqueología y la Antropología Cultural han tenido especial importancia para el respeto y mantenimiento de construcciones y objetos materiales del pasado. 
entorno físico, homo hábilis es para algunos antropólogos más importante que sapiens. Vivir no es sólo pensar, vivir es hacer más allá de las exigencias del instinto vinculándonos a la realidad con una visión diferente. En este caso para hacer algo material previamente debemos pensar, ya que planificar interiormente es una forma de pensamiento, pero los problemas que tenemos que resolver no se solucionan tan sólo pensando sino, de una manera u otra, trasladando esos pensamientos a objetos reales. Los objetos materiales que se mantienen a lo largo del tiempo son tangibles en cuanto pueden ser apreciados partiendo de los sentidos. Durante mucho tiempo patrimonio cultural se ha identificado con objetos de diversa índole de este tipo para cuya preservación se requiere también de acciones materiales.

Pero también es posible crear objetos no materiales que responden a formas de vida del pasado como leyendas, mitos, música, fiestas, danzas que, con las variaciones del caso, se mantienen en el presente sustentados por la tradición y que sin tener la firmeza de los materiales, son más vitales y portadores de un espíritu que sobrepasa a la captación sensorial ${ }^{10}$. Es evidente que estas realidades son parte viviente de la identidad cultural ya que parten de una dimensión interna con mayor contenido que lo material. Este tipo de tradiciones vivientes son más acordes con la condición humana, que por naturaleza es cambiante como cultura. Las realizaciones no materiales que persisten hasta nuestros días -no como relatos sino como prácticas- han incorporado los cambios de diversa

10 Lo que denominamos memoria colectiva tiene más peso en este tipo de manifestaciones culturales ya que están viabilizadas por el traslado, sobre todo verbal, de los que tratan de mantener para el futuro expresiones menos sustentadas en creaciones materiales. 
índole que necesariamente se dan con el tiempo, sin que ello implique la renuncia o pérdida del espíritu. Incorporar a medios electrónicos músicas tradicionales no acaban con la tradición, el ser y sentir está más allá de las variaciones técnicas de la expresión.

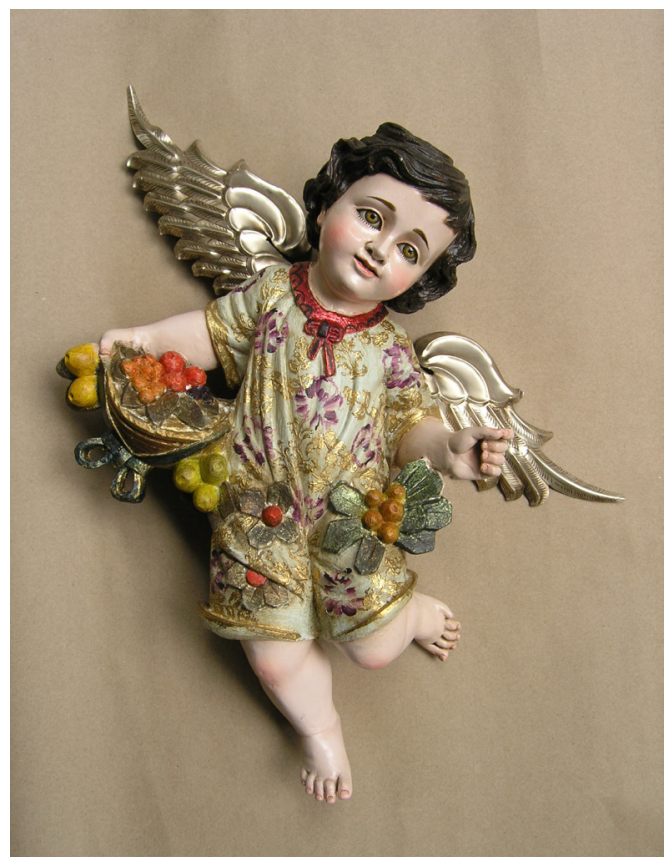

Los avances tecnológicos son parte de la evolución cultural, de los que no es posible prescindir. Decir que las nuevas tecnologías atentan contra la identidad, tiene su parte de verdad, pero muy grave sería pretender mantener el espíritu y la tradición dando de espaldas a los cambios técnicos propios de nuestra temporalidad; parte del cambio y la tradición radica en la capacidad de adaptación a las nuevas circunstancias y tradiciones de la existencia, lo que es factible en varios aspectos de la vida en común. 
La vida colectiva es tan variable y compleja que, a primera vista, es muy difícil establecer cuáles de esas manifestaciones culturales deben ser consideradas patrimonio cultural inmaterial en cada cultura y aquellas que merecen tener categoría universal. La UNESCO intenta una definición. La decisión se puntualizó en la "Convención para la Salvaguardia del Patrimonio Cultural Inmaterial" Reunida en París el 17 de Octubre de 2003". En ese documento dice:

"Se entiende por Patrimonio Cultural Inmaterial, los usos, representaciones, expresiones, conocimientos técnicas-Junto con los instrumentos, objetos, artefactos y espacios culturales que les son inherentes- que las comunidades, los grupos y en algunos casos los individuos, reconozcan como parte integrante de su patrimonio cultural. Este patrimonio cultural inmaterial que se transmite de generación en generación, es recreado constantemente por las comunidades y grupos en función de su entorno, su interacción con la naturaleza y su historia, infundiéndoles un sentimiento de identidad y continuidad y contribuyendo así a promover el respeto de la diversidad cultural y la creatividad humana. A los efectos de la presente convención se tendrá en cuenta únicamente el Patrimonio Cultural Inmaterial que sea compatible con los instrumentos internacionales de derechos humanos existentes y con los imperativos de respeto mutuo entre comunidades, grupos, individuos y desarrollo sostenible".

El Patrimonio Cultural Inmaterial, según se define en el párrafo 1 supra, se manifiesta en particular en los ámbitos siguientes:

a) Tradiciones y expresiones orales incluido el idioma como vehículo del Patrimonio Cultural Inmaterial;

b) Artes del espectáculo,

c) Usos sociales, rituales y actos festivos,

d) Conocimientos y usos relacionados con la naturaleza y el universo.

e) Técnicas artesanales tradicionales". 
Se entiende por Salvaguardia las medidas destinadas a garantizar la viabilidad del Patrimonio Cultural Inmaterial, comprendidas la identificación, documentación, investigación, preservación, protección, promoción, valorización y transmisión -básicamente a través de la enseñanza formal y no formal- y revitalización del Patrimonio en sus distintos aspectos".

El sentido temporal y colectivo de estas formas de patrimonio es parte de su estructura interna. Es frecuente que cuando en los sectores populares se pregunta a alguien la razón por la que se siguen haciendo determinadas cosas de esa manera, la respuesta sea "porque así lo hacían nuestros mayores". En las comunidades prácticamente no hay disposiciones formales que obligan a realizar determinadas gestiones, espontáneamente asumen responsabilidades personas de reconocido prestigio, al margen de las funciones que tengan.

Jóvenes aprenden los procedimientos y las creencias se mantienen colectivamente, de manera que siempre hay nuevas personas de nuevas generaciones que se incorporan a la tradición y se encargan de relevar a los que dejaron este mundo. En algunos casos, como las técnicas artesanales, el factor familiar suele jugar un importante papel.

Vista esta problemática desde afuera, no es fácil comprender por qué los mantenedores de estas tradiciones, sobre todo de las fiestas, dedican tanto tiempo y esfuerzo en mengua, con frecuencia, de sus intereses económicos con ocurre con los priostes. Si podemos hablar de recompensa, se encuentra en el prestigio y respetabilidad que ganan entre los integrantes de la comunidad, hecho que no es posible cuantificarlo monetariamente. 


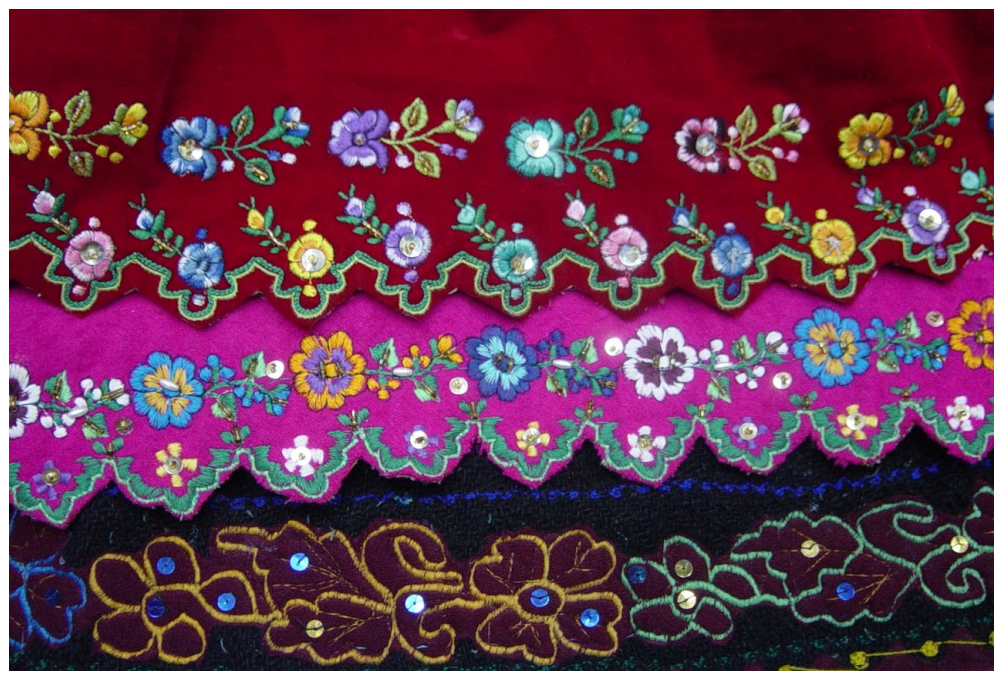

La identidad que parte de la cultura popular, puesta de manifiesto en estas formas de comportamiento, es un sólido nexo de unión entre las personas, que supera las distancias espaciales. Migrantes que se han establecido en países con mejores recursos económicos, no renuncian sino, con frecuencia, robustecen estas manifestaciones de identidad con alto contenido solidario ${ }^{11}$.

Un muy elevado porcentaje del patrimonio cultural inmaterial se encuentra en los sectores populares. En los últimos años ha habido un cambio en la actitud de las instituciones gubernamentales, que se esmera en

11 En nuestro medio hay casos de migrantes sobre todo a Estados Unidos, que buscan ser priostes de celebraciones tradicionales y se trasladan a los lugares correspondientes para financiar con esplendor estas fiestas, demostrando su pertenencia a la comunidad pese a la distancia. Quizás también para demostrar que fueron exitosos en la "aventura" que emprendieron.

26 
mantenerlo y apoyarlo. En un cercano pasado, partiendo del dudoso presupuesto de que educar es incorporar al pueblo a las manifestaciones civilizadas de la cultura elitista, la actitud solía ser de desprecio a estas manifestaciones consideradas como expresiones de ignorancia.

Hoy se considera que uno de los deberes del Estado es preservar y mantener la identidad a la que oficialmente hay que apoyarla y respetarla incorporándola como parte del proceso educativo. El patrimonio está fundamentado en el pasado, pero las acciones y actitudes del presente, individuales y gubernamentales, son muy importantes para su subsistencia y proyección al futuro. El sentido de temporalidad no sólo está presente en el patrimonio en sí sino en la visión de las personas y del sector público. 


\section{Bibliografía consultada}

Álvarez González, Francisco

Las Exclusivas del Hombre, 2003, Universidad de Cuenca, Cuenca.

Cordero Î́niguez, Juan

Signos de Identidad Cuencana, 2011, Municipalidad de Cuenca, Cuenca.

Jaramillo Paredes, Mario

Estudio Histórico sobre Ingapirca, 1976, Pontificia Universidad Católica del Ecuador, Quito.

Malo González, Claudio

Arte y Cultura Popular, 2006, Universidad del Azuay CIDAP Cuenca.

Monteforte, Mario

Los Signos del Hombre, 1985, Pontificia Universidad Católica del Ecuador Sede en Cuenca, Cuenca.

Real Academia Española

Diccionario de la lengua española, 2001, Espasa Calpe, Madrid.

Redfield, Robert

The Little Comunity Peasanta Society and Culture, 1963, The University of Chicago Press, Chicago.

Rhee Kyu Ho

Struggle for National Identity in the Third World, 1983, Hollym Inrternational Corp, Seul.

UNESCO

Claves del Siglo XXI, 2002, Crítica, Madrid. 